04,09

\title{
Обнаружение дефектов, связывающих свободные поляроны в окрашенных щелочно-галоидных кристаллах
}

\author{
(C) E.B. Коровкин \\ Институт физики твердого тела РАН, \\ Черноголовка, Россия \\ E-mail: Korovkin@issp.ac.ru
}

(Поступила в Редакцию 22 марта 2016 г.

В окончательной редакции 20 июля 2016 г.)

\begin{abstract}
Сделано предположение, что дефектами, связывающими свободные поляроны в окрашенных щелочногалоидных кристаллах (ЩГК), являются $\mathrm{F}^{\prime}$-центры, то есть дефекты, тормозящие движение дислокаций (фотопластический эффект). Выполненные эксперименты подтвердили это предположение. Таким образом, анионная вакансия в ЩГК может при низкой температуре удерживать на себе три электрона - два электрона на глубоком уровне ( $\mathrm{F}^{\prime}$-центр) и еще один электрон в виде связанного поляронного состояния. Он удерживается благодаря выигрышу в энергии при взаимодействии локальной деформации полярона и локальной деформации, окружающей $\mathrm{F}^{\prime}$-центр, несмотря на наличие кулоновского отталкивания.
\end{abstract}

DOI: $10.21883 /$ FTT.2017.03.44157.107

Ранее, используя двухступенчатую фотопроводимость, в окрашенных гамма-облучением кристаллах ЩГК при $T<78 \mathrm{~K}$ был обнаружен и исследован интересный объект [1-4], который по своим характеристикам был определен как полярон, а именно:

1. В NaCl (сперва исследовался этот кристалл) энергия термического разрушения, максимум оптического поглощения (ОП) и максимум фотоионизации (ФИ) (фоторазрушения) относились как $1: 2: 3$, что соответствовало теории Пекара для поляронов [5]. Правда, для исследованных позднее кристаллов $\mathrm{KCl}$ и $\mathrm{KBr}$ это соотношение выполнялось хуже.

2. В теоретической работе [6], посвященной исследованию ОП малого полярона, показано, что спектр ОП полярона представляет собой широкий пик, изрезанный более узкими равноотстоящими фононными пиками, положение которых на шкале энергий отвечает формуле $E=n \cdot E_{p}$, где $E_{p}-$ энергия продольного фонона. Наши экспериментальные результаты находятся в хорошем согласии с этим [1,2]. Единственное расхождение заключается в том, что полуширина пика ОП примерно в 2 раза меньше, чем в этой теории. Теория [6] предсказывает уменьшение амплитуды фононных осцилляций при повышении температуры, что также подтверждается в наших экспериментах.

3. Последовательности фононных осцилляций в ОП и в ФИ позволяют с большой точностью определить величину энергии продольных оптических фононов, проявляющихся в этих спектрах. При этом, величина фонона из спектров ФИ оказывается больше таковых из спектров ОП как в $\mathrm{NaCl}$, так в $\mathrm{KCl}$ и в $\mathrm{KBr}$ на $10.8 \%, 7.4 \%$ и $5.2 \%$ соответственно $[1,3,4]$, что подтверждает результат теоретических работ $[7,8]$, в которых утверждается, что должно наблюдаться „смягчение“ фононных мод полярона по сравнению с продольным оптическим фононом кристалла.
Таким образом, несомненно, этот объект является поляроном, но так как при низких температурах он стабилен и может быть создан в большой концентрации (достигалось ОП в $1 / \mathrm{cm}$ ), то это связанный полярон. Быть может, именно этот факт, отличающий его от поляронов теории [6], и приводит к тому, что полуширина его спектра ОП примерно в 2 раза меньше, чем в этой теории.

В некоторых теориях ОП поляронов, например $[9,10]$, обычно имеется некая бесфононная линия при некоторой энергии $E_{0}$ (не всегда наблюдаемая в экспериментах) и фононная структура (пики или ступеньки), подчиняющиеся формуле $E=E_{0}+n \cdot E_{p}$. Отличительной особенностью теории [6] является $E_{0}=0$. Это очень сильное отличие и то, что оно подтверждается в наших экспериментах, указывает на корректность принятых в этой теории упрощений.

Теоретической разработкой оптического поглощения малого полярона много занимался Александров с соавторами $[7,8,11$ и др.]. В работе [11] гораздо больше параметров, чем в [6]. В результате только при определенных значениях этих параметров спектр ОП выглядит так, как в экспериментальной работе [1]. Положение фононных осцилляций одного из этих спектров (стр. 250, рис. $1, \lambda=2.5$ ) также неплохо соответствует формуле $E=n \cdot E_{p}$, а период их следования подтверждает „смягчение“ фононных мод поляронов на $40 \%$, на что указывалось в других работах этих авторов $[7,8]$. Важным достоинством работы [11] является также то, что отношение положения максимума ОП к полуширине спектра ОП в этой теории при определенных значениях параметров превышает значение 1.5 (в эксперименте около двух), в то время как в работе [6] это отношение близко к 1.0 .

Далее представляется важным определить, на каких дефектах кристалла связывается этот полярон. При 
этом возникает интересная гипотеза - не является ли $\mathrm{F}^{\prime}$-центр такой ловушкой для полярона? Известно $[12,13,14]$, что $\mathrm{F}^{\prime}$-центр является стопором для движущейся дислокации (фотопластический эффект). Этот эффект в окрашенных ЩГК невелик. Следовательно, это не очень сильный стопор и искажения решетки вокруг него не столь велики, что может обеспечить достаточно нежный захват полярона, чтобы образовавшийся связанный полярон не сильно отличался от свободного.

Для проверки этой гипотезы были проведены следующие эксперименты. Они выполнялись на автоматизированной установке по измерению двухступенчатой (фотоиндуцированной) фотопроводимости (ФП). Образцы монокристаллического номинально чистого (содержание примесей меньше $10^{-3 \%}$ ) $\mathrm{NaCl}$ размером $4 \times 4 \times 10 \mathrm{~mm}$ облучались гамма-облучением до дозы $5 \cdot 10^{6} \mathrm{rad}$. Такой образец помещался в оптический гелиевый криостат в вертикальном положении между двумя электродами и охлаждался до $T=20 \mathrm{~K}$. В качестве источника света использовалась лампа накаливания СИ-8, имеющая ленточный вертикально расположенный излучатель, изображение которого проектировалось на образец при помощи линзы диаметром $80 \mathrm{~mm}$. Спектр света изменялся при помощи стеклянных светофильтров $80 \times 80 \mathrm{~mm}$.

Экспериментально можно было организовать три различных состояния кристалла по отношению к концентрации $\mathrm{F}^{\prime}$-центров.

a) После охлаждения в темноте в кристалле нет $\mathrm{F}^{\prime}$-центров.

b) Экспозиция образца светом в F-полосу (F-свет, фильтры СС8 и С3C14) в течение достаточно длительного времени (в наших экспериментах 300-600 s) создает, вследствие сильного перекрытия F- и $\mathrm{F}^{\prime}$-полос, динамически равновесное состояние с достаточно большой концентрацией $\mathrm{F}^{\prime}$-центров.

c) Экспозиция образца оранжевым светом (фильтр OC14, длина волны света $580-2700 \mathrm{~nm}$ ) в течение $300 \mathrm{~s}$ и более переводит образец в другое динамически равновесное состояние с гораздо меньшей концентрацией $\mathrm{F}^{\prime}$-центров.

Таким образом, чередуя эти экспозиции, можно многократно переводить образец из состояния $(b)$ в (c) и обратно. Перевести же образец из этих состояний в состояние (a) можно только отогревом образца с последующим охлаждением его в темноте.

Каждое измерение состояло из трех последовательных шагов:

1-й шаг - „установка прибора на нуль“. Экспозицией ИК-светом (фильтры ИКС3 + ИКС1) в течение $10 \mathrm{~s}$ уничтожались все ИК-ловушки (связанные поляроны) в образце, если они были.

2-й шаг - „окрашивание или маркировка имеющихся в образце ловушек для связанных поляронов“. Небольшой дозой F-света (интенсивность - 0.1 от максимальной, экспозиция $2 \mathrm{~s}$ ) осуществлялся выброс с F-центров порции электронов. Они заселяли имеющиеся в образце

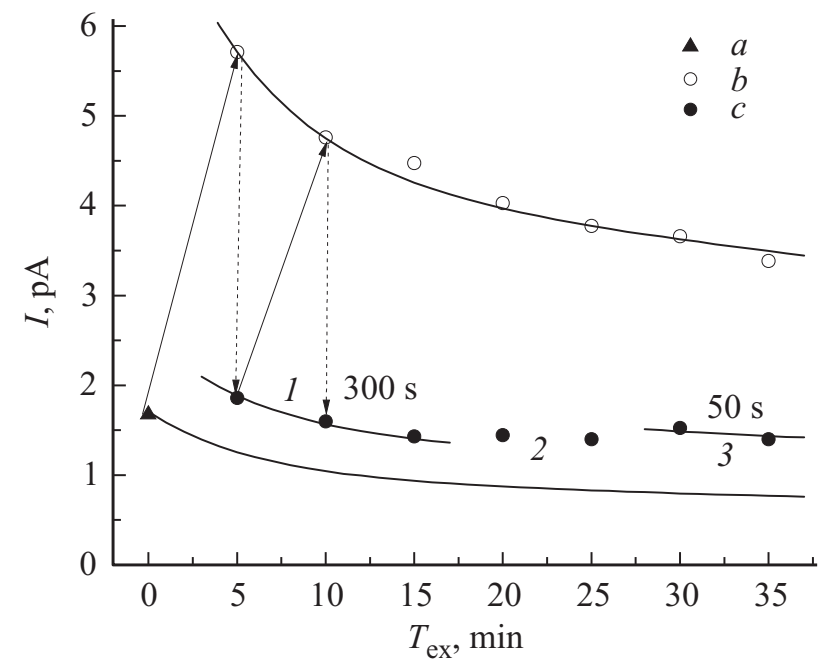

Рис. 1. Влияние различной световой обработки на концентрацию поляронных ловушек. $a-$ сигнал без световой обработки; $b-$ F-свет, $300 \mathrm{~s} ; c-$ оранжевый свет (OC14): $1-300 \mathrm{~s}, 2-200$ и $150 \mathrm{~s}, 3-50 \mathrm{~s}$; стрелка вверх и вправо - обработка F-светом 300 s; стрелка вниз - обработка оранжевых светом $300 \mathrm{~s}$.

ловушки, образуя связанные поляроны в количестве, пропорциональном концентрации этих ловушек.

3-й шаг - измерение ИК-фотопроводимости, вызываемой наличием в образце связанных поляронов. Сигнал пропорционален концентрации поляронов и, следовательно, концентрации поляронных ловушек в образце.

Таким образом, если гипотеза верна, то состоянию $(b)$ будет соответствовать большой сигнал ФП по сравнению с таковым в состоянии $(c)$. Результат представлен на рис. 1. По оси абсцисс отложено время F-световой экспозиции. Точке $(a)$ соответствует сигнал ФП при отсутствии начальной световой обработки (состояние (a) образца). Наличие сигнала ФП означает, что и в этом состоянии в образце присутствует некоторое количество мелких электронных ловушек (не обязательно поляронных), опустошаемых ИК-светом. Далее, после 5-минутной экспозиции F-светом (стрелка вверх и вправо), переводящей образец в состояние $(b)$, получаем большой сигнал ФП, указывающий на резкое увеличение поляронных ловушек. Затем экспозицией оранжевым светом в течении $300 \mathrm{~s}$ образец переводился в состояние $(c)$ (стрелка вниз), что приводило к резкому уменьшению сигнала ФП (уменьшение концентрации поляронных ловушек). Далее эти эксперименты можно было повторять многократно, что и демонстрирует рисунок. Таким образом, большей концентрации $\mathrm{F}^{\prime}$-центров соответствует бо́льшая концентрация поляронных ловушек, что подтверждает гипотезу о том, что $\mathrm{F}^{\prime}$-центр является эффективной ловушкой для поляронов.

Известно, что в окрашенных ЩГК имеются, кроме F-центров, более сложные электронные центры окраски: M-, R-центры и т.д. в сторону их усложнения. Область 
чувствительности их к свету сдвигается по мере их усложнения в красную и далее в ближнюю ИК-область. В обычном состоянии они, подобно F-центру, имеют нейтральное зарядовое состояние и не создают вокруг себя локальной деформации кристалла. Но захватив электрон, они, подобно $\mathrm{F}^{\prime}$-центру, деформируют вокруг себя решетку кристалла и могут, таким образом, стать ловушками для поляронов. Естественно возникает вопрос не являются ли и они ловушками для поляронов? Дело в том, что полоса пропускания ОС14 простирается от 580 до $2700 \mathrm{~nm}$. Следовательно, эти центры накапливаются и разрушаются синхронно с F'-центрами. Поэтому, чтобы выяснить роль этих ловушек, был выполнен следующий эксперимент:

Сперва выбиралась подходящая длительность экспозиции (фильтр ОС14, в дальнейшем от КС10 до КС19) такой, чтобы выйти из уровня динамического равновесия и, таким образом, сделать сигнал более чувствительным к изменению экспозиции или спектра разрушающего ловушки света. Рис. 1 демонстрирует это. Точкам (1) соответствует длительность экспозиции оранжевого света $-300 \mathrm{~s}$, точкам (2) - 200 и $150 \mathrm{~s}$, точкам (3) $-50 \mathrm{~s}$. Видно, что только при экспозиции $50 \mathrm{~s}$ сигнал надежно отличается от динамически равновесного (точки 1).

Из рис. 1 (а также и рис. 2) видно, что точки $(b)$ не укладываются на прямую. Причина этого заключается в том, что концентрации F-центров в образце уменьшаются под действием F-света. Это обстоятельство приводит к тому, что порция электронов, выбрасываемая в образец под действием F-света (второй шаг прцедуры измерения), также не остается постоянной, а уменьшается, что и приводит к уменьшению чувствительности измерений по некому закону. Кривая, проходящая через точки $(b)$ и демонстрирующая этот закон, представляет сумму двух экспонент. Медленная экспонента $(t=250-300 \mathrm{~min})$ соответствует обычному для гамма-облученных ЩГК постепенному обесцвечиванию кристалла под действием света. Быстрая - уменьшение концентрации F-центров из-за перераспределения электронов с них под действием F-света на электронные центры, которые не могут удерживать дополнительные электроны при комнатной температуре, но хорошо удерживают их при низкой. При отогреве образца эта часть электронов частично возвращается на F-центры. Сигнал ФП от неких, уже имеющихся в образце ловушек (точка $(a)$ ), изменяется также по этому закону, что и демонстрирует кривая, исходящая из этой точки. То, что точки $(c)$ находятся выше этой кривой, как раз и означает невозможность полностью разрушить оранжевым светом все $\mathrm{F}^{\prime}$-центры, о чем упоминалось выше. Аналогичным образом проведены кривые через точки 1 и точки 3.

Далее, для выяснения роли „красных“ центров был выполнен эксперимент, результаты которого представлены на рис. 2. Точки (b) (пустые кружки, величина $A$ ) представляют результат измерения ФП после 300 секундной экспозиции F-светом, а точки $(c)$ (черные кружки, величина $B)$ - сигнал ФП после по-

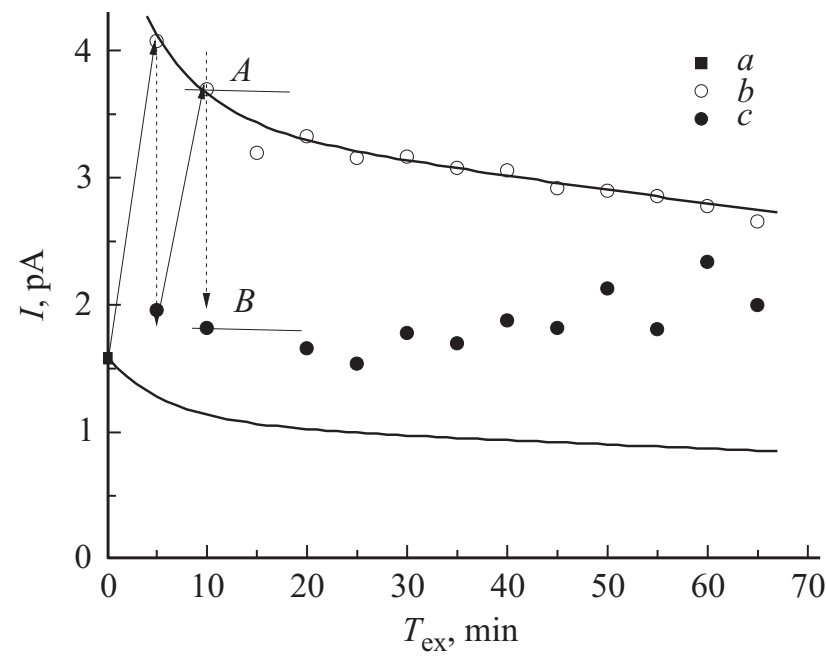

Рис. 2. Влияние света различного спектра на разрушение поляронных ловушек. $a-$ сигнал без световой обработки; $b-$ F-свет, $300 \mathrm{~s}$; $c$ - свет через различные светофильтры, последовательно: JC14, ОС14, КС10, КС13, КС14, КС15, $\mathrm{KC} 17, \mathrm{KC} 18, \mathrm{KC} 17, \mathrm{OC} 14, \mathrm{KC} 19$.

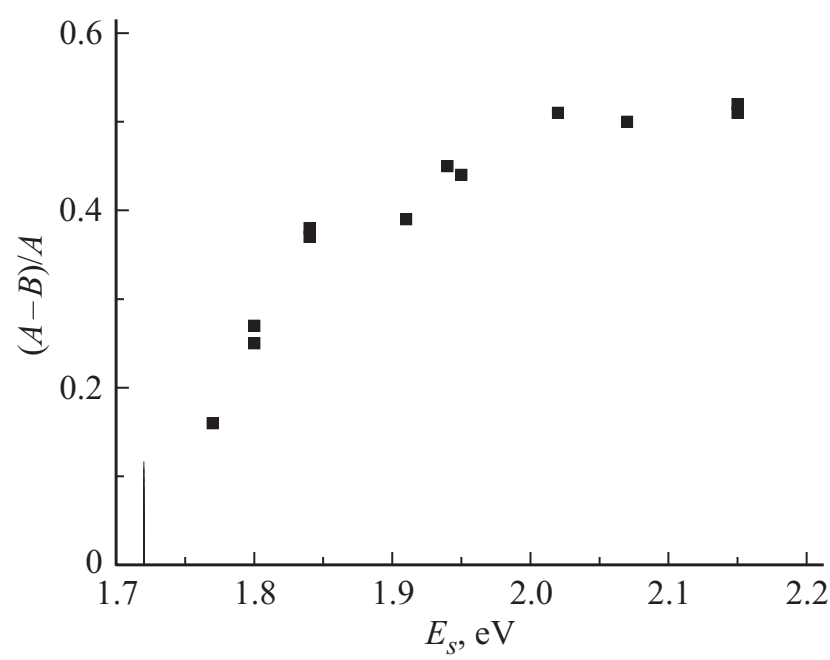

Рис. 3. Спектральная зависимость фоторазрушения поляронных ловушек. $E_{s}$ - положение коротковолнового края полосы пропускания фильтров на уровне 0.5 , вертикальная линия при $E_{s}=1.72 \mathrm{eV}$ - длинноволновый край спектра $\mathrm{F}^{\prime}$-центров [15].

следующей за этим 50 секундной экспозиции разрушающим поляронные ловушки светом разного спектрального состава (разные светофильтры, от ОС14 к КС10 и далее до КС19, смещающие полосу спектра последовательно в длинноволновую сторону). Полоса пропускания этих фильтров начинается от $E_{s}$ (на уровне 0.5$)$ и продолжается в сторону меньших энергий до $L=2700 \mathrm{~nm}$. Величина $E_{s}$ изменялась от $2.2 \mathrm{eV}$ для $\mathrm{OC} 14$ и до $1.8 \mathrm{eV}$ для КС19.

Относительная глубина разрушения $(A-B) / A$ дана на рис. 3 в зависимости от $E_{s}$. Видно резкое падение этой величины при смещении в длинноволновую сторону. Это 
указывает на то, что среди создаваемых F-светом поляронных ловушек присутствуют в основном ловушки, разрушаемые светом в области $L<680 \mathrm{~nm}$, т.е. роль „красных“ центров мала или исключена вовсе.

Таким образом, $\mathrm{F}^{\prime}$-центр может удерживать еще один электрон в виде связанного полярона, несмотря на наличие кулоновского отталкивания. Он удерживается благодаря выигрышу в энергии при взаимодействии локальной деформации полярона и локальной деформации, окружающей $\mathrm{F}^{\prime}$-центр, на некотором расстоянии от $\mathrm{F}^{\prime}$-центра. В результате образуется сферическая область (возможно сферическая полость) вокруг $\mathrm{F}^{\prime}$-центра, в которой полярон может двигаться аналогично свободному полярону в кристалле, проявляя тем самым сходные со свободным поляроном свойства. Это приводит к далеко идущим следствиям. Например, свойства свободного полярона, трудно экспериментально измеряемые из-за его короткого времени жизни, не должны сильно отличаться от таковых для связанного полярона. Это дает для глубины залегания поляронного уровня (зоны) в $\mathrm{NaCl}$ величину в $0.162 \mathrm{eV}$, что глубже залегания уровня возбужденного F-центра $(0.08-0.15 \mathrm{eV})$. В результате электрон с возбужденного F-центра может уходить в кристалл в виде свободного полярона при сколь угодно низкой температуре (в работе [14] при $T=1.6 \mathrm{~K}$ ). А спад фотопроводимости при понижении температуры в районе $70 \mathrm{~K}$ объясняется не только термоактивированным выбросом электронов с возбужденного F-центра в зону проводимости, а также тем, что $\mathrm{F}^{\prime}$-центры при $T<70 \mathrm{~K}$ являются для поляронов (по полупроводниковой терминологии) эффективными ловушками, а при $T>70 \mathrm{~K}$ - центрами прилипания.

\section{Выводы}

1. Гипотеза, что связывающими поляроны ловушками являются те самые центры окраски, которые создаются при освещении образца F-светом и приводят к фотопластическому эффекту, т. е. $\mathrm{F}^{\prime}$-центры, подтверждена.

2. Кроме $\mathrm{F}^{\prime}$-центров, возникающих при экспозиции образца F-светом, в кристалле имеется небольшое количество электронных ловушек, захватывающих электроны и теряющих их под действием ИК-света. Вопрос создаются ли при этом на них связанные поляронные состояния - остается открытым.

3. Роль электронных центров окраски, теряющих электроны под действием красного света $(L>700 \mathrm{~nm})$, в связывании свободных поляронов мала или отсутствует вовсе.

4. Выводы теоретической работы [6], а также [11] при некоторых величинах имеющихся в ней параметров, хорошо согласуются с экспериментом [1-4]. А именно: в вопросах положения максимума ОП, его полуширины, наличия системы равноотстоящих фононных осцилляций, положения этой системы относительно начала оси энергий и ее зависимости от температуры, а также наличия „смягчения“ фононных мод поляронного образования по отношению к величине обычного продольного оптического фонона.

5. Даны качественные объяснения причины такого согласия, наличия эффективного ухода электронов с возбужденных F-центров в кристалл (наблюдается фотопластический эффект при $1.6 \mathrm{~K}$ [14], а также участия $\mathrm{F}^{\prime}$-центров в формировании наблюдаемого спада ФП при охлаждении в районе $70-80 \mathrm{~K}$.

\section{Список литературы}

[1] Е.В. Коровкин, Т.А. Лебедкина. ФТТ 33, 10, 2822 (1991).

[2] Е.В. Коровкин, Т.А. Лебедкина. ФТТ 35, 3, 642 (1993).

[3] Е.В. Коровкин, Т.А. Лебедкина. ФТТ 37, 11, 3536 (1995).

[4] Е.В. Коровкин, Т.А. Лебедкина. ФТТ 42, 8, 1412 (2000).

[5] С.И. Пекар, ЖЭТФ 16, 341 (1946); ЖЭТФ 18, 525 (1948); Избранные труды. Наукова думка, Киев (1988). Гл. 2. С 76.

[6] J. Loos, J. Straka. Czech. J. Phys. B 39, 316 (1989).

[7] A.S. Alexandrov, H. Capellman. Phys. Rev. B 43, 2042 (1991).

[8] A.S. Alexandrov, V.V. Kabanov, D.K. Ray. Phys. Rev. B 49, 14, 9915 (1994)

[9] J. Devreese, R. Evard, E. Kartheuser. Solid State Commun. 7, 767 (1969).

[10] E. Kartheuser, R. Evard, J. Devreese. Phys. Rev. Lett. 22, 3, 94 (1969).

[11] A.S. Alexandrov, V.V. Kabanov, D.K. Ray. Physica C 224, 247 (1994).

[12] J. Nadeau. J. Appl. Phys. 35, 669, (1964).

[13] Г.А. Ермаков, Е.В. Коровкин, Я.М. Сойфер. ФТТ 16, 3, 697 (1974).

[14] Е.В. Коровкин. Письма в ЖЭТФ 35, 11, 481 (1982).

[15] D.W. Lynch, D.A. Robinson. Phys. Rev. 174, 1050 (1968). 